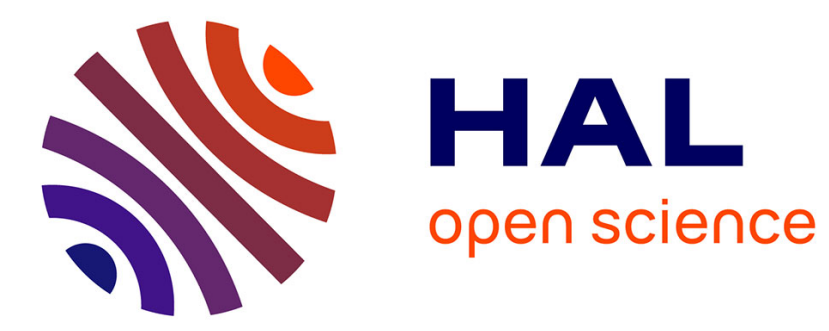

\title{
Instabilities and waves on a columnar vortex in a strongly stratified and rotating fluid
}

\author{
Junho Park, Paul Billant
}

\section{To cite this version:}

Junho Park, Paul Billant. Instabilities and waves on a columnar vortex in a strongly stratified and rotating fluid. Physics of Fluids, 2013, 25 (8), pp.086601. 10.1063/1.4816512 . hal-00996422

\section{HAL Id: hal-00996422}

https://hal-polytechnique.archives-ouvertes.fr/hal-00996422

Submitted on 27 May 2014

HAL is a multi-disciplinary open access archive for the deposit and dissemination of scientific research documents, whether they are published or not. The documents may come from teaching and research institutions in France or abroad, or from public or private research centers.
L'archive ouverte pluridisciplinaire HAL, est destinée au dépôt et à la diffusion de documents scientifiques de niveau recherche, publiés ou non, émanant des établissements d'enseignement et de recherche français ou étrangers, des laboratoires publics ou privés. 


\title{
Instabilities and waves on a columnar vortex in a strongly stratified and rotating fluid
}

\author{
Junho Park ${ }^{\mathrm{a})}$ and Paul Billant \\ Laboratoire d'Hydrodynamique (LADHYX), CNRS, Ecole Polytechnique, \\ Palaiseau Cedex F-91128, France
}

(Received 18 December 2012; accepted 28 May 2013; published online 1 August 2013)

\begin{abstract}
This paper investigates the effect of the background rotation on the radiative instability of a columnar Rankine vortex in a strongly stratified fluid. We show that a cyclonic background rotation strongly stabilizes the radiative instability. The modes become neutral when the Rossby number Ro is below a critical value which depends on the azimuthal wavenumber of the wave. In the limit of small Rossby number, there exist fast neutral waves that are not captured by the quasi-geostrophic theory. In the presence of anticyclonic background rotation, the centrifugal instability dominates the radiative instability only when $-400 \lesssim R o<-1$. The numerical stability analysis is completed by asymptotic analyses for large wavenumbers which explain the properties and mechanisms of the waves and the instabilities. The stability of a continuous smoothed Rankine vortex is also investigated. The most amplified azimuthal wavenumber is then finite instead of infinite for the Rankine vortex. (c) 2013 AIP Publishing LLC. [http://dx.doi.org/10.1063/1.4816512]
\end{abstract}

\section{INTRODUCTION}

In 1880, Lord Kelvin has described the waves sustained by a Rankine vortex. ${ }^{1}$ These waves, now called the vortex Kelvin waves, are an important subject in vortex dynamics. ${ }^{2,3}$ In homogeneous fluids, the Kelvin waves are confined to the vortical region of the vortex and belong to the family of inertial waves. In contrast, vortex waves are no longer confined to the vortex core in stratified fluids but can exist outside in the form of internal gravity waves. A subtle coupling between the vortex rotation and internal waves leads to an instability which spontaneously emits internal waves from the vortex.

This radiative instability does not require the presence of vorticity and can occur on the potential flow around a rotating cylinder. ${ }^{4,5}$ The instability mechanism has been explained in terms of wave over-reflection by means of a Wentzel-Kramers-Brillouin-Jeffreys (WKBJ) analysis. ${ }^{6,7}$ A wave emitted from the vortex core is indeed over-reflected at the critical radius where the angular velocity of the vortex is equal to the azimuthal phase velocity of the vortex. This phenomenon has been also explained in terms of pseudo-angular momentum. The transmitted wave has a negative pseudoangular momentum so that the reflected wave should have a larger amplitude to conserve the total pseudoangular momentum. ${ }^{8-10}$ The radiative instability occurs not only in stratified fluids, ${ }^{6-10}$ but also in shallow-water ${ }^{11}$ or in compressible fluids with the emission of acoustic waves. ${ }^{12}$

Geophysical flows are not only stably stratified but also under the influence of the planetary rotation. In this paper, we shall see that the radiative instability becomes much weaker in the presence of a cyclonic background rotation. Such stabilization has been already reported by Schecter and Montgomery ${ }^{9}$ for some particular modes and vortex profiles. Here, we shall investigate systematically the effect of the background rotation on all the discrete waves sustained by a Rankine vortex and a smoothed Rankine vortex. We shall see that the stabilization of the radiative instability depends

\footnotetext{
a) junho.park@ladhyx.polytechnique.fr
} 
strongly on the azimuthal wavenumber of the waves and on the vortex profile. These results will be explained theoretically by means of a WKBJ analysis.

In the presence of small anticyclonic background rotation, a vortex is unstable to the centrifugal instability but we shall show that the radiative instability remains the most dangerous instability when the Rossby number $R o=2 \Omega / f$ is sufficiently large in absolute value (i.e., $R o \lesssim-400$ ) where $\Omega$ is the angular velocity at the vortex center and $f$ is the Coriolis parameter.

The regime $-1<R o<0$ where the centrifugal instability no longer exists has been already considered. ${ }^{13}$ We have shown that the radiative instability can also occur in this range if the azimuthal wavenumber is larger than 2 . We shall therefore restrict the analysis here to the range $1 / R o>-1$ (i.e., $f>-2 \Omega$ ).

The paper is organized as follows: the stability problem is described in Sec. II. In Sec. III, the effect of cyclonic rotation is presented. In Sec. IV, WKBJ analyses for large vertical wavenumber $k$ are performed to explain the instability mechanism and properties. The effect of an anticyclonic rotation is investigated in Sec. V. Finally, the effect of the smoothness of the vorticity profile is presented in Sec. VI.

\section{PROBLEM FORMULATION}

We consider an axisymmetric vortex with velocity components $\left(0, U_{\theta}(r), 0\right)$ in a cylindrical coordinate system $(r, \theta, z)$ which is rotating about the vertical axis at angular velocity $\Omega_{b}=f / 2$ where $f$ is the Coriolis parameter. The fluid is assumed to be inviscid and stably stratified with a constant Brunt-Väisälä frequency $N$. We subject this vortex to infinitesimally small three-dimensional perturbations of velocity $\tilde{\mathbf{u}}=\left(\tilde{u}_{r}, \tilde{u}_{\theta}, \tilde{u}_{z}\right)$, pressure $\tilde{p}$ and density $\tilde{\rho}$, written in the form

$$
(\tilde{\mathbf{u}}, \tilde{p}, \tilde{\rho})=(\mathbf{u}(r), p(r), \rho(r)) e^{-i \omega t+i k z+i m \theta}+c . c,
$$

where c.c denotes the complex conjugate, $\omega$ is the complex frequency, $k$ is the vertical wavenumber, and $m$ is the azimuthal wavenumber. Under the Boussinesq approximation, the linearized equations of momentum, density conservation, and continuity for the perturbations read

$$
\begin{gathered}
i s u_{r}-\left(f+\frac{2 U_{\theta}}{r}\right) u_{\theta}=-\frac{d}{d r}\left(\frac{p}{\rho_{0}}\right), \\
i s u_{\theta}+(f+\zeta) u_{r}=-\frac{i m p}{\rho_{0} r}, \\
i s u_{z}=-i k \frac{p}{\rho_{0}}-\frac{g}{\rho_{0}} \rho, \\
i s \rho=N^{2} \frac{\rho_{0}}{g} u_{z}, \\
\frac{1}{r} \frac{d}{d r}\left(r u_{r}\right)+\frac{i m}{r} u_{\theta}+i k u_{z}=0,
\end{gathered}
$$

where $s=-\omega+m U_{\theta} / r$ is the Doppler-shifted frequency, $\zeta=\frac{1}{r} \frac{d}{d r}\left(r U_{\theta}\right)$ is the axial vorticity of the vortex, $g$ is the gravity, and $\rho_{0}$ is a constant reference density. From Eqs. (2)-(6), a single equation for the pressure $p$ can be deduced

$$
\frac{d^{2} p}{d r^{2}}+\left(\frac{1}{r}-\frac{\Delta^{\prime}}{\Delta}\right) \frac{d p}{d r}+\left[-\frac{k^{2}}{N^{2}-s^{2}} \Delta-\frac{m^{2}}{r^{2}}+\frac{m \Delta}{r s}\left(\frac{f+\frac{2 U_{\theta}}{r}}{\Delta}\right)^{\prime}\right] p(r)=0,
$$

where $\Delta(r)=(f+\zeta)\left(f+\frac{2 U_{\theta}}{r}\right)-s^{2}$ and prime denotes differentiation with respect to $r{ }^{13,14}$ Equations (7) and (1) imply the symmetry $\omega(k, m)=\omega(-k, m)=-\omega^{*}(-k,-m)$, where the asterisk denotes the complex conjugate. Hence, we consider hereafter only positive $k$ and $m$. Moreover, we assume that the fluid is strongly stratified so that the hydrostatic approximation can be applied: $N \gg|s|$ (i.e., $N^{2}-s^{2} \approx N^{2}$ ). This assumption implies that there is no singularity where $|s|=N$. In addition, (7) depends only on $k$ and $N$ through the rescaled vertical wavenumber $\tilde{k}=\frac{k}{N}$ owing to the self-similarity of strongly stratified fluids. 
As a base flow, we first consider the Rankine vortex: $U_{\theta}(r)=\Omega r$ for $r<R$ and $U_{\theta}(r)$ $=\Omega R^{2} / r$ for $r>R$, where $R$ is the radius of the vortex core, and then a smoothed Rankine vortex in Sec. VI. We impose the boundary conditions that the perturbations are nonsingular at the vortex center $r=0$ and decay exponentially or radiate energy outward as $r \rightarrow \infty$. For the Rankine vortex, we also have to apply the kinematic and dynamic boundary conditions at the boundary of the vortex core $r=R$, which enforce the continuity of the radial velocity and the pressure: $u_{r}\left(R^{-}\right)=u_{r}\left(R^{+}\right)$, $p\left(R^{-}\right)=p\left(R^{+}\right)$. By expressing $u_{r}$ in terms of $p$ by means of (2) and (3), these boundary conditions lead to the dispersion relation

$$
\frac{p^{\prime}\left(R^{+}\right)}{p\left(R^{+}\right)}=\frac{\Delta\left(R^{+}\right)}{\Delta\left(R^{-}\right)} \frac{p^{\prime}\left(R^{-}\right)}{p\left(R^{-}\right)}-\frac{m(f+2 \Omega)}{R s(R)}\left[1-\frac{\Delta\left(R^{+}\right)}{\Delta\left(R^{-}\right)}\right] .
$$

The non-singular solution of (7) inside the vortex core $r<R$ is

$$
p(r) \sim I_{m}\left(\tilde{k} r \sqrt{(f+2 \Omega)^{2}-(-\omega+m \Omega)^{2}}\right),
$$

where $I_{m}$ is the modified Bessel function of the first kind of order $m$. When $\omega$ is purely real, $I_{m}$ increases exponentially with $r$ when $|f+2 \Omega|>|-\omega+m \Omega|$ and is wavelike when $|f+2 \Omega|<$ $|-\omega+m \Omega|$. Outside the vortex core $r>R$, (7) needs to be integrated numerically. The integration is performed inward starting far outside the vortex core $r \gg R$ from the asymptotic solution which satisfies the boundary condition for $r \rightarrow \infty$,

$$
p(r) \sim H_{m}^{(1)}\left(\tilde{k} r \sqrt{\omega^{2}-f^{2}}\right),
$$

where $H_{m}^{(1)}$ is the Hankel function of the first kind of order $m$. For real $\omega, H_{m}^{(1)}$ behaves as an outgoing wave when $\omega>|f|$, while it decreases exponentially with $r$ when $|\omega|<|f|$. Starting from an initial guess value for $\omega$, the secant method is used to find the values of $\omega$ which satisfy the dispersion relation (8).

\section{NUMERICAL RESULTS}

\section{A. Cyclonic rotation}

Figure 1 shows the frequency $\omega_{r}$ and growth rate $\omega_{i}$ of the first three branches for $m=1$ for different non-negative values of the Coriolis parameter $f$. The results found previously for $f / \Omega=0$ are shown to serve as a reference. ${ }^{6}$ The frequency of the first branch starts from the $2 \mathrm{D}$ dispersion relation $\omega=(m-1) \Omega=0$ at zero vertical wavenumber $k$ independently of $f$. As $f$ increases, the frequency increases with $k$ more rapidly but it always tends to $\Omega$ as $k \rightarrow \infty$ regardless of $f$. The corresponding growth rate $\omega_{i}$ remains also maximum for a finite $k$ but the maximum growth rate
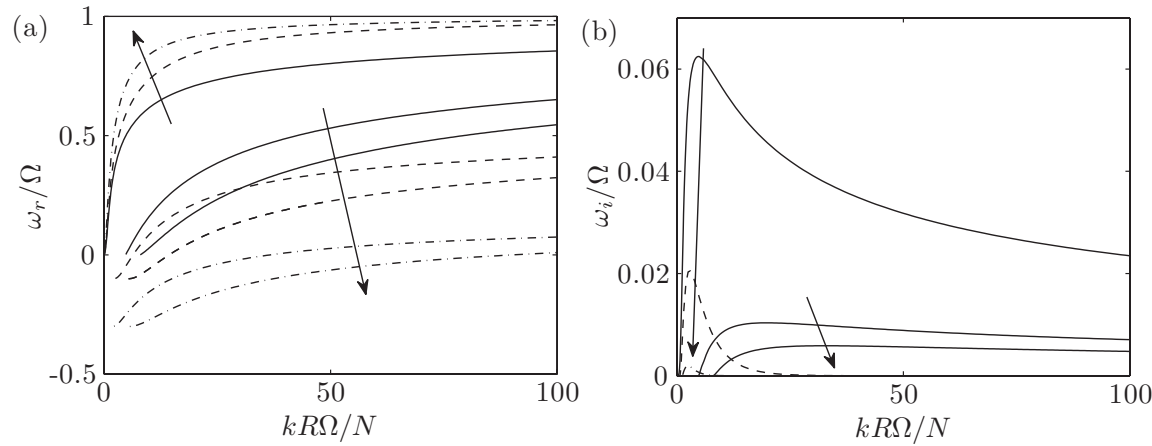

FIG. 1. (a) Frequency and (b) growth rate as a function of the rescaled vertical wavenumber $k R \Omega / N$ for $m=1$ for different Coriolis parameters: $f / \Omega=0$ (solid lines), $f / \Omega=0.1$ (dashed lines), and $f / \Omega=0.3$ (dashed-dotted lines). Only the first three branches are shown. $f$ increases in the direction of the arrows. For $f / \Omega=[0.1,0.3]$, growth rates of the higher branches are not visible because they are too small. 


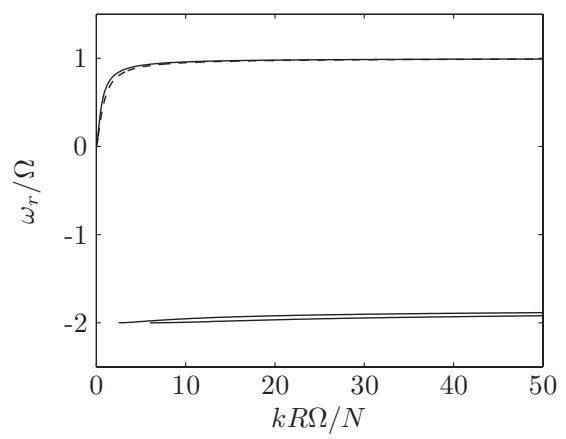

FIG. 2. Frequency of the first three branches for $f / \Omega=2$ and $m=1$. Solid lines are numerical results. The dashed line shows the quasi-geostrophic dispersion relation (11).

decreases rapidly as $f$ increases. We have found that the maximum growth rate becomes of order of $O\left(10^{-5} \Omega\right)$, i.e., negligibly small when $f \gtrsim 0.5 \Omega$. In contrast to the first branch, the frequencies of the next branches start around $\omega=-f$ at non-zero $k$ and tend to $\omega_{r}=\Omega-\sqrt{f(f+2 \Omega)}$ as $k \rightarrow \infty$. The growth rates of the next branches are much smaller than for the first branch and they decrease more rapidly as $f$ increases. For example, the maximum growth rate $\omega_{i}$ of the second branch becomes of order $O\left(10^{-5} \Omega\right)$ as soon as $f \gtrsim 0.05 \Omega$.

Figure 2 shows the frequency of the first three branches for $m=1$ when $f / \Omega$ is larger: $f / \Omega=2$. The frequency of the branches still lies in the same range: $0<\omega_{r}<\Omega$ for the first branch and $-f<\omega_{r}<\Omega-\sqrt{f(f+2 \Omega)}$ for the next branches but the growth rate is now strictly zero. As seen in Figure 2, the frequency of the first branch is well predicted by the dispersion relation of the Rankine vortex in the quasi-geostrophic limit (i.e., $f \gg \Omega$ ), ${ }^{15}$

$$
\omega=m \Omega\left[1-2 I_{m}\left(\frac{k R f}{N}\right) K_{m}\left(\frac{k R f}{N}\right)\right]
$$

where $I_{m}$ and $K_{m}$ are the modified Bessel functions of order $m$. In contrast, the next branches have a frequency of order $|f|$. Hence, they are not captured by the quasi-geostrophic dispersion relation (11) because quasi-geostrophy assumes $O(|\omega|,|\Omega|) \ll|f|$.

Figure 3 displays some examples of pressure eigenfunctions. We clearly see a radiating inertiagravity wave train outside the vortex core for the eigenmode of the first branch (Figures 3(a) and $3(\mathrm{~b})$ ). However, the amplitude of this radiating wave is attenuated when $f$ increases from $f / \Omega=0$ to $f / \Omega=0.3$. In contrast, the pressure eigenfunction of the second branch for $f / \Omega=0.3$ (Figure 3(c)) has only one node outside the vortex core and no radiating wavetrain. The next branches are similar except that the number of nodes outside the vortex core is $n-1$, where $n$ is the branch number. These distinct properties will be explained in Sec IV.
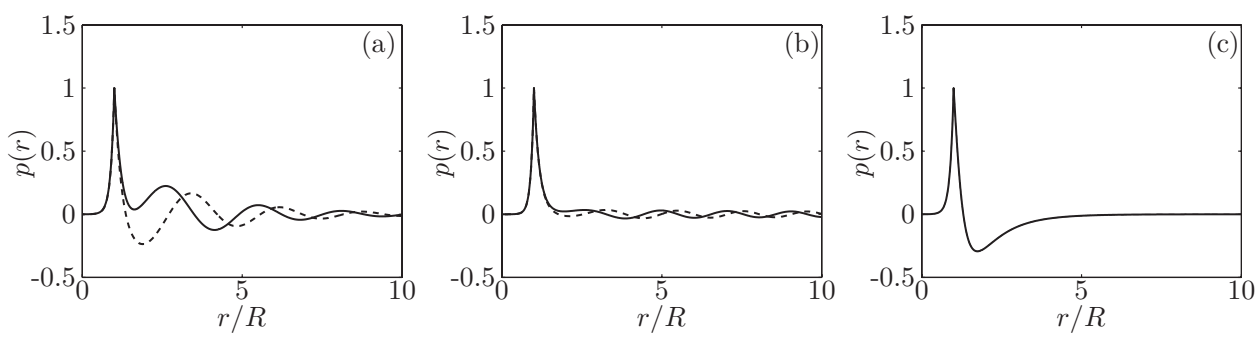

FIG. 3. Pressure eigenfunction for $k R \Omega / N=5$ and (a) $f / \Omega=0$, (b) $f / \Omega=0.3$ for the first branch, and (c) $f / \Omega=0.3$ for the second branch. Solid and dashed lines represent the real and imaginary parts, respectively. 

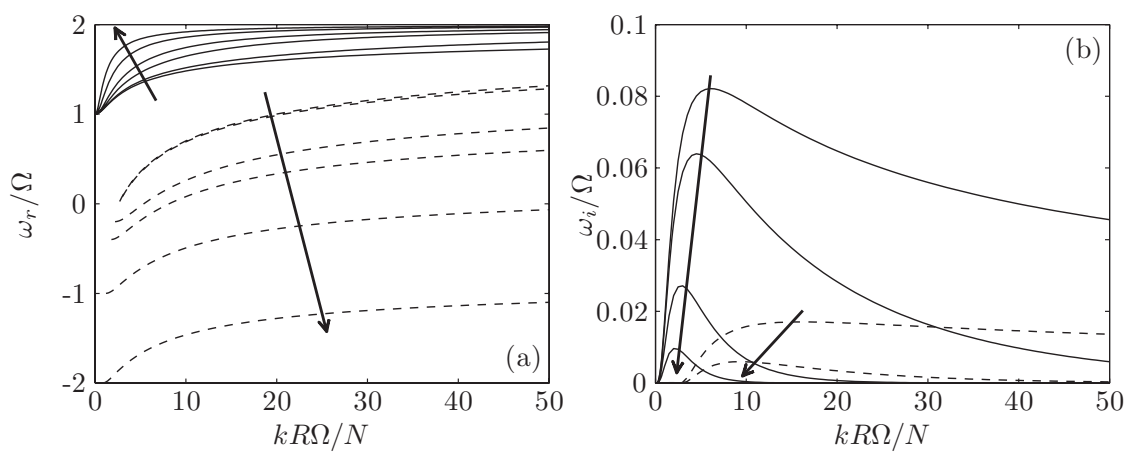

FIG. 4. Similar to Fig. 1 but for $m=2$, (a) frequency $\omega_{r}$ and (b) growth rate $\omega_{i}$ for $m=2$ for the first branch (solid lines) and the second branch (dashed lines) for $f / \Omega=[0,0.04,0.2,0.4,1,2] . f / \Omega$ increases in the direction of the arrows.

\section{B. Variation with the azimuthal wavenumber}

Figure 4 shows the frequency and growth rate for $m=2$. When $f$ varies, the frequency of the first branch now lies in the range $[\Omega, 2 \Omega]$, while the frequency of the next branches lies in the range $-f<\omega_{r}<2 \Omega-\sqrt{f(f+2 \Omega)}$. More generally, we have found for arbitrary non-zero azimuthal wavenumber $m$ that the frequency of the first branch lies in the range

$$
(m-1) \Omega \leq \omega_{r}<m \Omega,
$$

while the frequency range of the next branches is

$$
-f<\omega_{r}<m \Omega-\sqrt{f(f+2 \Omega)} .
$$

The particular case of the axisymmetric perturbation $m=0$ is treated in the Appendix.

The maximum growth rate of the first branch for $m=2$ and for $f=0$ is $\omega_{i} \approx 0.08 \Omega$ (for $k R \Omega / N$ $\approx 6)$ and is slightly larger than for $m=1\left(\omega_{i} \approx 0.06 \Omega\right.$ for $\left.k R \Omega / N \approx 5\right)$. Actually, for larger $m$, the maximum growth rate continues to increase monotonically with $m$ but the corresponding eigenmode concentrates near $r=R$. Following Ref. 16, an asymptotic analysis can be performed for $m \gg 1$ by introducing the rescaled variables: $\bar{r}=m(r / R-1), \omega_{0}=\omega / \Omega-m, k_{1}=k R \Omega /(N m)$, and $\bar{f}=f / \Omega$ where $\left(\bar{r}, \omega_{0}, k_{1}, \bar{f}\right)$ will be assumed to be of order one. Then (7) for $r>R$ becomes at leading order in $m$,

$$
\frac{d^{2} p}{d \bar{r}^{2}}+\bar{\Delta} \frac{d}{d \bar{r}}\left(\frac{1}{\bar{\Delta}}\right) \frac{d p}{d \bar{r}}+\left[-k_{1}^{2} \bar{\Delta}-1-\frac{4(\bar{f}+2)}{\bar{\Delta}}\right] p(\bar{r})=O\left(\frac{1}{m}\right),
$$

where $\bar{\Delta}(\bar{r})=\bar{f}(\bar{f}+2)-\left(\omega_{0}+2 \bar{r}\right)^{2}$. This equation is equivalent to the one obtained by Ref. 16 for the radial velocity. It has to be solved numerically except for $f=0$ where an analytical solution exists

$$
p(\bar{r})=C_{0}\left(\bar{r}+\frac{\omega_{0}}{2}\right) D_{-\frac{1}{2}-\frac{i}{4 k_{1}}}\left(2 \sqrt{k_{1}}\left(\bar{r}+\frac{\omega_{0}}{2}\right) e^{-i \frac{\pi}{4}}\right),
$$

where $D_{v}$ denotes the parabolic cylinder function of order $v$ and $C_{0}$ is a constant. Note that (15) matches the outgoing wave boundary condition (10) as $\bar{r} \rightarrow \infty$.

In Figure 5, we show the rescaled frequency $\omega_{r} / \Omega-m$ and the growth rate $\omega_{i}$ as a function of the rescaled vertical wavenumber $k R \Omega /(\mathrm{Nm})$ for $f / \Omega=0$ and $f / \Omega=0.2$. We see that the numerical results tend to the asymptotic results (bold lines) as $m$ increases for both values of $f / \Omega$. The maximum growth rate for $f=0$ is approximately $\omega_{i} \approx 0.1 \Omega$ and is reached around $k R \Omega /(N m) \approx 2$. These values are very close to those obtained in the case of the potential flow around a rotating cylinder. ${ }^{16}$ This indicates that the potential flow of the Rankine vortex is responsible for the instability, whereas the vortex core plays no active role. However, the frequency range for the Rankine vortex (Figure 5(a)) and the flow around a rotating cylinder is different since the first branch is absent in the latter flow owing to the impermeable boundary condition at $r=R$. 

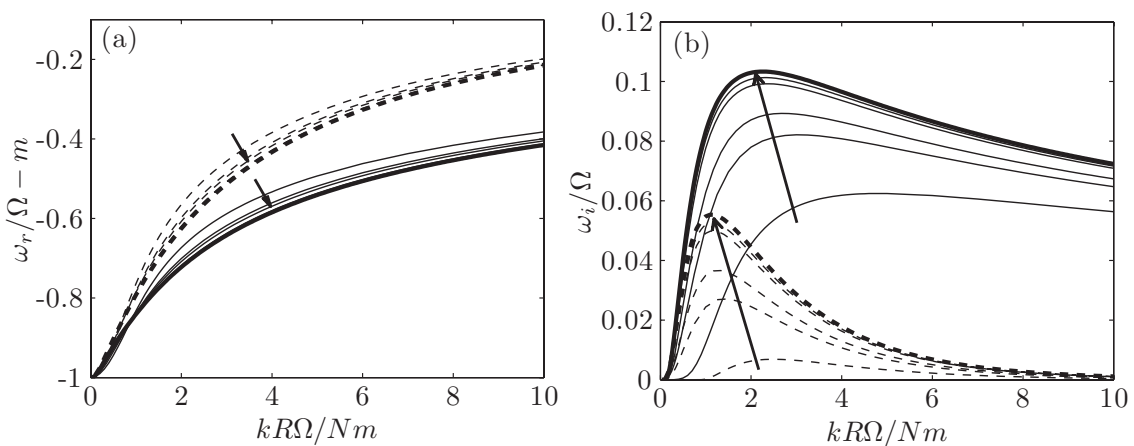

FIG. 5. (a) Rescaled frequency $\omega_{r} / \Omega-m$ and (b) growth rate $\omega_{i}$ of the first branch as a function of rescaled vertical wavenumber $k R \Omega /(\mathrm{Nm})$ for $m=[1,2,3,10,20]$ and for $f / \Omega=0$ (solid lines) and $f / \Omega=0.2$ (dashed lines). Bold lines have been obtained from the large $m$ asymptotic equation (14). $m$ increases in the direction of the arrows.

Figure 6 summarizes the effect of a cyclonic background rotation on the radiative instability: the maximum growth rate is plotted as a function of the Coriolis parameter $f$ for different azimuthal wavenumbers $m$. We see that the maximum growth rate $m=\infty$ (bold line) quickly decreases as $f$ increases but remains positive whatever $f$. We can remark that the maximum growth rate tends to the asymptotic maximum growth rate (bold line) as $m$ increases. For finite $m$, the growth rate goes to zero at a particular value of $f / \Omega$.

\section{WKBJ ANALYSIS}

\section{A. Formulation}

The numerical results obtained in Sec. III can be explained by means of a WKBJ analysis for large rescaled vertical wavenumber $\tilde{k} \cdot{ }^{6,7,17}$ For large $\tilde{k}$, Eq. (7) becomes at leading order

$$
\frac{d^{2} p}{d r^{2}}+\left(\frac{1}{r}-\frac{\Delta^{\prime}}{\Delta}\right) \frac{d p}{d r}+\left[-\tilde{k}^{2} \Delta+O(1)\right] p(r)=0
$$

The WKBJ approximation focuses on rapid exponential behaviours of the solution ${ }^{18}$ so that the $O(1)$ term in (16) can be neglected at leading order. The WKBJ approximation of the solution of (16) is

$$
p(r) \sim \frac{(-\Delta)^{1 / 4}}{r^{1 / 2}}\left[A \exp \left(i \tilde{k} \int_{r_{t}}^{r} \sqrt{-\Delta} d t\right)+B \exp \left(-i \tilde{k} \int_{r_{t}}^{r} \sqrt{-\Delta} d t\right)\right],
$$

where $A, B$ are constants and $r_{t}$ is a turning point where $\Delta\left(r_{t}\right)=0$. If the eigenfrequency $\omega$ is predominantly real (i.e., $\omega_{r} \gg \omega_{i}$ ) and $\Delta<0$, the approximation (17) is wavelike. Conversely if

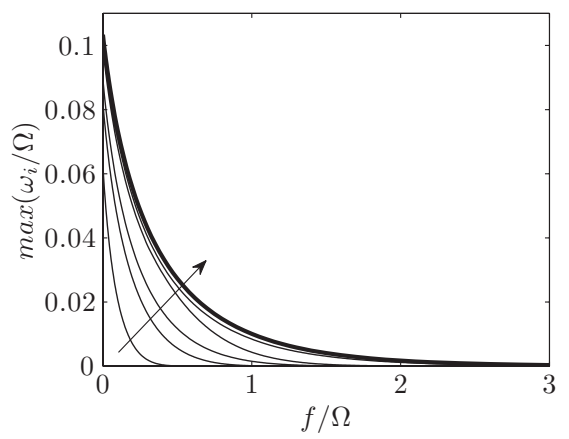

FIG. 6. Maximum growth rate as a function of the Coriolis parameter for different azimuthal wavenumbers. Thin and thick solid lines represent numerical results for $m=[1,2,3,10,20]$ and asymptotic results for $m \gg 1$, respectively. $m$ increases in the direction of the arrow. 
$\Delta>0$, the WKBJ approximation has an exponential behaviour and (17) can be rewritten

$$
p(r) \sim \frac{\Delta^{1 / 4}}{r^{1 / 2}}\left[C \exp \left(-\tilde{k} \int_{r_{t}}^{r} \sqrt{\Delta} d t\right)+D \exp \left(\tilde{k} \int_{r_{t}}^{r} \sqrt{\Delta} d t\right)\right],
$$

where $C$ and $D$ are constants. The first term in (18) decays exponentially with $r$, whereas the second term increases with $r$. Regarding the wavelike solution (17), we can define the phase velocity $v_{\phi}$ and the group velocity $v_{g}$ in the radial direction of each term. For the first term, they are

$$
v_{\phi}=\frac{\omega_{r}}{l_{r}}=\frac{\omega_{r}}{\tilde{k} \operatorname{Re}(\sqrt{-\Delta})}, v_{g}=\frac{\partial \omega_{r}}{\partial l_{r}}=\frac{\operatorname{Re}(\sqrt{-\Delta})}{\tilde{k} \operatorname{Re}(-s)},
$$

where $l_{r}=\operatorname{Re}\left[\frac{\partial}{\partial r}\left(\tilde{k} \int_{r_{t}}^{r} \sqrt{-\Delta} d t\right)\right]$ is the local radial wavenumber. The sign of the phase velocity $v_{\phi}$ depends on the sign of the frequency $\omega_{r}$, while the sign of the group velocity $v_{g}$ depends on the sign of the Doppler-shifted frequency $s$. This implies that the group velocity $v_{g}$ changes its sign at the critical point $r_{c}$ where $s\left(r_{c}\right)=0$. As $r \rightarrow \infty$, the group velocity is $v_{g}=\sqrt{\omega_{r}^{2}-f^{2}} /\left(\tilde{k} \omega_{r}\right)$. Therefore, when $\omega_{r}>f>0$, the first term in (17) represents an outgoing wave, whereas the second term corresponds to an incoming wave.

The WKBJ approximations (17) and(18) are valid except at the turning point $r_{t}$ where $\Delta\left(r_{t}\right)=0 .{ }^{18}$ In the neighborhood of the turning point $r_{t}$, (7) approximates at leading order to

$$
\frac{\mathrm{d}^{2} p}{\mathrm{~d} \tilde{r}^{2}}-\frac{1}{\tilde{r}} \frac{\mathrm{d} p}{\mathrm{~d} \tilde{r}} \mp \tilde{r} p=O(\epsilon)
$$

where $\epsilon=\frac{1}{\left( \pm \Delta^{\prime}\left(r_{t}\right) \tilde{k}^{2}\right)^{1 / 3}}$ and $\tilde{r}=\frac{r-r_{t}}{\epsilon}$. The sign \pm corresponds to the sign of $\Delta^{\prime}\left(r_{t}\right)$. The solution of (20) is

$$
p(\tilde{r})=a_{ \pm} A i^{\prime}( \pm \tilde{r})+b_{ \pm} B i^{\prime}( \pm \tilde{r}),
$$

where $a_{+}, a_{-}, b_{+}, b_{-}$are constants, Ai, Bi denote Airy's functions. ${ }^{19}$ From the asymptotic behaviour of the Airy functions for $\tilde{r} \rightarrow+\infty$ and $\tilde{r} \rightarrow-\infty$, the WKBJ approximations in the regions $r>r_{t}$ and $r<r_{t}$ can be matched. ${ }^{18}$

\section{B. Radial structure of the WKBJ approximations}

To determine the sign of $\Delta$ and the location of the turning point $r_{t}$, it is useful to define the epicyclic frequencies ${ }^{17}$

$$
\omega_{ \pm}(r)=m \frac{U_{\theta}}{r} \pm \sqrt{(f+\zeta)\left(f+\frac{2 U_{\theta}}{r}\right)},
$$

for which $\Delta=0$. When the frequency $\omega_{r}$ lies in the interval $\omega_{-}<\omega_{r}<\omega_{+}, \Delta$ is positive so that the WKBJ approximations are exponential. On the other hand, $\Delta$ is negative when $\omega_{r}>\omega_{+}$or $\omega_{r}$ $<\omega_{-}$, and the WKBJ approximations are wavelike.

Figure 7 shows two examples of the epicyclic frequencies for two different values of $f / \Omega$ for $m=1$. They are typical of all the configurations that can be encountered for any azimuthal wavenumber $m \geq 1$ in the case of a cyclonic rotation. We have also plotted the critical frequency $\omega_{c}(r)=$ $m U_{\theta} / r$ with dashed lines to indicate where the group velocity changes its sign. Since the epicyclic frequencies always decrease with $r$ for $r>R$, we may distinguish three different ranges of frequencies:

(I): $\max \left(\omega_{+}(\infty), \omega_{-}\left(R^{+}\right)\right)<\omega_{r}<\omega_{+}\left(R^{+}\right)$

(II): $\min \left(\omega_{+}(\infty), \omega_{-}\left(R^{+}\right)\right)<\omega_{r}<\max \left(\omega_{+}(\infty), \omega_{-}\left(R^{+}\right)\right)$,

(III): $\quad \omega_{-}(\infty)<\omega_{r}<\min \left(\omega_{+}(\infty), \omega_{-}\left(R^{+}\right)\right)$.

The frequency ranges $\omega_{r}>\omega_{+}\left(R^{+}\right)$or $\omega_{r}<\omega_{-}(\infty)$ are not considered because there is no eigenfunction. Indeed, the outer solution is wavelike for any $r>R$ but an outgoing WKBJ approximation (first term in (17)) can never satisfy the dispersion relation (8). 

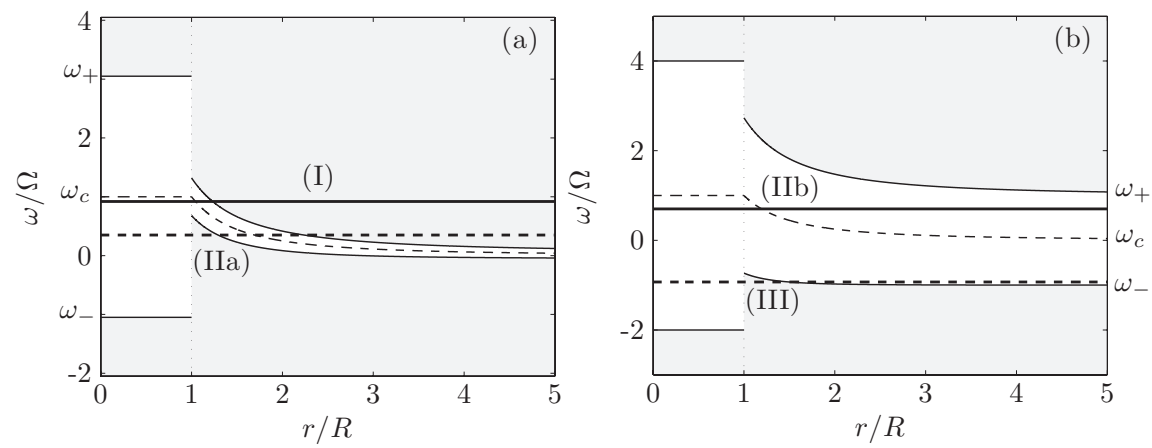

FIG. 7. Epicyclic frequencies $\omega_{+}$and $\omega_{-}$(thin solid lines) and critical frequency (thin dashed lines) as a function of $r$ for (a) $f / \Omega=0.05$ and (b) $f / \Omega=1$ for $m=1$. Dotted lines represent the boundary of the vortex core. Thick solid and dashed lines show examples of the frequency $\omega_{r}$ for the four different configurations (I, IIa, IIb, III). The regions where the WKBJ approximations are wavelike (i.e., $\Delta<0$ ) are shaded.

In the frequency range (I), there is a single turning point $r_{t 2}$ where the frequency $\omega$ is equal to $\omega=\omega_{+}\left(r_{t 2}\right)$, and the WKBJ approximations are wavelike for $r>r_{t 2}$ and exponential-like for $r<r_{t 2}$ (see the example shown by a bold solid line in Figure 7(a)). The configuration in the frequency range (II) depends on $\omega_{+}(\infty)$ and $\omega_{-}\left(R^{+}\right)$. When $\omega_{-}\left(R^{+}\right)>\omega_{+}(\infty)$ (configuration hereafter referred as IIa), there are two turning points $r_{t 2}$ and $r_{t 1}$ such that $\omega=\omega_{-}\left(r_{t 1}\right)=\omega_{+}\left(r_{t 2}\right)$. The WKBJ approximations are exponential-like between $r_{t 1}$ and $r_{t 2}$, and wavelike for $r>r_{t 2}$ and $r<r_{t 1}$ (see the thick dashed line in Figure 7(a)). Note that the critical point $r_{c}$ is enclosed between $r_{t 1}$ and $r_{t 2}$. In contrast, when $\omega_{-}\left(R^{+}\right)<\omega_{+}(\infty)$ (case hereafter referred as IIb), there exists no turning point and the WKBJ approximations are exponential-like for any $r>R$ as for the example displayed by a bold solid line in Figure 7(b). Finally, in the range (III), there is a single turning point $r_{t 1}$ and the WKBJ approximations are exponential-like for $r>r_{t 1}$ and wavelike for $r<r_{t 1}$ (see the example illustrated by a thick dashed line in Figure 7(b)).

In Figure 8, we have plotted the frequencies $\omega_{+}\left(R^{+}\right), \omega_{+}(\infty), \omega_{-}\left(R^{+}\right)$, and $\omega_{-}(\infty)$ as a function of the Coriolis parameter $f$. The type of configurations is indicated in each domain ${ }^{6,7}$ has shown that the radiative instability can exist when there exists two distinct wave regions, i.e., for the configuration (IIa). In this case, an incident wave (i.e., with positive group velocity) is reflected at the turning point $r_{t 1}$ and transmitted to an outgoing wave for $r>r_{t 2}$ through the exponential region which is analogous to a potential barrier. However, because the group velocity is reversed at the critical point $r_{c}$ in the potential barrier, the reflected wave for $r<r_{t 1}$ has a larger amplitude than the incident wave. This phenomenon of over-reflection is the origin of the radiative instability. A condition for the existence of the radiative instability is therefore that the configuration (IIa) exists,

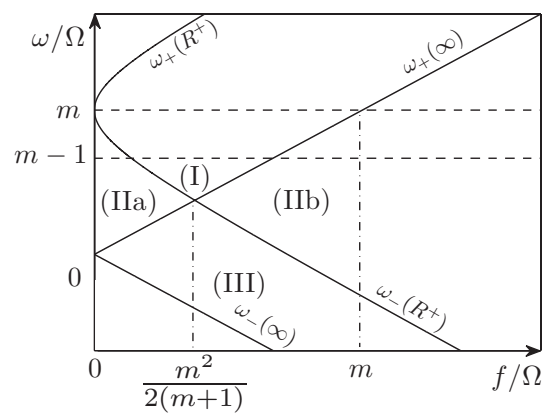

FIG. 8. Characteristic frequencies as a function of $f / \Omega$. The labels refer to the structure of the WKBJ approximations described in the text. The dashed lines delimit the frequency range of the first branch $[(m-1) \Omega, m \Omega]$. The dashed-dotted lines show the thresholds for the radiative instability of the first and next branches. 
i.e., $\omega_{-}\left(R^{+}\right)>\omega_{+}(\infty)$ (see Figure 8$)$ giving

$$
m \Omega-\sqrt{f(f+2 \Omega)}>f,
$$

or in terms of the Rossby number $R o$,

$$
R o \equiv 2 \Omega / f>\frac{4(m+1)}{m^{2}} .
$$

However, we shall see in Sec. IV C that this condition is only sufficient but not necessary because the first branch is particular for the Rankine vortex.

We now derive in details the WKBJ approximations for each of the four possible configurations: (I), (IIa), (IIb), (III).

\section{One turning point: Case (I)}

In the configuration (I), there is one turning point $r_{t 2}$ and the WKBJ approximations are wavelike for $r>r_{t 2}$. In order to impose that the wave is outgoing for $r \rightarrow \infty$, we put $B=0$ in (17). Then, the matching WKBJ approximation in the region $r<r_{t 2}$ is

$$
p(r) \sim A \mathrm{e}^{-\frac{\mathrm{i} \pi}{4}} \frac{\Delta^{1 / 4}}{r^{1 / 2}}\left[\frac{1}{2} \mathrm{e}^{-\tilde{k} \int_{r}^{r_{2}} \sqrt{\Delta(t)} d t}+i \mathrm{e}^{\tilde{k} \int_{r}^{r_{2} 2} \sqrt{\Delta(t)} d t}\right] .
$$

This approximation can be directly inserted in the dispersion relation (8). We can also further expand the dispersion relation in powers of $\tilde{k}$ to obtain explicitly the eigenfrequency. This gives at leading order

$$
\omega_{r}=m \Omega-\frac{\alpha}{R \tilde{k}}, \omega_{i}=\frac{\alpha^{2}}{2 m R \Omega} \sqrt{f(f+2 \Omega)} \frac{W}{\tilde{k}},
$$

where $\alpha=2 m \Omega /(f+\sqrt{f(f+2 \Omega)})$ and $W=\exp \left(-2 \tilde{k} \int_{R}^{r_{12}} \sqrt{\Delta(t)} d t\right)$. The frequency tends to $m \Omega$ as $\tilde{k} \rightarrow \infty$ and corresponds to the first branch. Surprisingly, its growth rate is positive and of order $O(W / \tilde{k})$ even if there is a single turning point. In fact, this first branch is particular because it originates from the vorticity jump present at $r=R$ in the Rankine vortex. This discontinuity acts like an infinitely small wave region which play the role of the second wave region needed for the radiative instability in the case of continuous vortex profiles. ${ }^{7}$

For this reason, the first branch is unstable as long as there exists an outer wave region, i.e., $\omega_{r}$ $>\min \left(\omega_{+}\right)=f$. Since the frequency range of the first branch is observed to be always in the range $[(m-1) \Omega, m \Omega]$, it is unstable for some frequency provided that $f<m \Omega$ (see Figure 8), i.e.,

$$
R o>\frac{2}{m} \text {. }
$$

The first branch is therefore unstable over a wider range of Rossby number than the next branches (see Eq. (24)).

\section{One turning point: Case (III)}

In the configuration (III), a turning point $r_{t 1}$ exists and the solution is now exponential-like for $r>r_{t 1}$. In order to impose the boundary condition at $r=\infty$, we put $D=0$ in (18). The matching WKBJ approximation in the region $r<r_{t 1}$ is then

$$
p(r) \sim \mathrm{e}^{\frac{\mathrm{i} \pi}{4}} \frac{(-\Delta)^{1 / 4}}{r^{1 / 2}}\left[A_{I 1} \mathrm{e}^{i \tilde{k} \int_{r}^{r_{1} 1} \sqrt{-\Delta(t)} d t}+A_{R 1} \mathrm{e}^{i \tilde{k} \int_{r}^{r_{r} 1} \sqrt{-\Delta(t)} d t}\right],
$$

where $A_{I 1}=C$ and $A_{R 1}=-i C$. By inserting (28) in (8), we obtain the asymptotic dispersion relation

$$
\tilde{k} \int_{R}^{r_{t 1}} \sqrt{-\Delta} d t+\frac{i}{4} \log (\mathcal{R})=n \pi
$$

where $n$ is the branch number and $\mathcal{R}$ is the reflection coefficient at $r_{t 1}$,

$$
\mathcal{R}=\mathcal{R}_{1} \equiv A_{R 1}^{2} / A_{I 1}^{2}=\mathrm{e}^{-i \pi} .
$$


As $\tilde{k} \rightarrow \infty$, the integral $\int_{R}^{r_{t 1}} \sqrt{-\Delta} d t$ should tend to zero in order to satisfy (29). This implies that the wave region concentrates near the vortex core $R$ as $\tilde{k} \rightarrow \infty$. By using this feature, we can express the eigenfrequency in powers of $\tilde{k}$,

$$
\begin{gathered}
\omega=m \Omega-\sqrt{f(f+2 \Omega)}-\beta /(\tilde{k} R \Omega)^{2 / 3}, \\
\beta=\left[\frac{3 \Omega^{2}}{\sqrt{2}}\left(n \pi-\frac{i}{4} \log (\mathcal{R})\right)(m \sqrt{f(f+2 \Omega)}-f)\right]^{2 / 3} / \sqrt{f(f+2 \Omega)},
\end{gathered}
$$

where $\mathcal{R}=\mathcal{R}_{1}$. Note that the frequency tends to $\omega_{r}=m \Omega-\sqrt{f(f+2 \Omega)}$ as $\tilde{k} \rightarrow \infty$ as observed for all the branches except the first one. Since the absolute value of the reflection coefficient $\left|\mathcal{R}_{1}\right|$ is unity (i.e., perfect reflection), $\beta$ is purely real so that the growth rate $\omega_{i}$ is zero. Hence, this configuration corresponds to neutral waves confined between $R$ and $r_{t 1}$.

\section{E. Two turning points: Case (lla)}

In the configuration (IIa), there are two turning points $r_{t 1}$ and $r_{t 2}$ (with $r_{t 1}<r_{t 2}$ ) enclosing a potential barrier. The WKBJ approximation for $r>r_{t 2}$ is an outgoing wave like in Subsection IV C. If the two turning points are well separated, the approximation for $r_{t 1}<r<r_{t 2}$ is also the same as in IV C. The WKBJ approximation which matches (25) for $r<r_{t 1}$ is then

$$
p(r)=\frac{(-\Delta)^{1 / 4}}{r^{1 / 2}}\left[A_{I 2} \mathrm{e}^{i \tilde{k} \int_{r}^{r_{11}} \sqrt{-\Delta(t)} d t}+A_{R 2} \mathrm{e}^{-i \tilde{k} \int_{r}^{r_{11}} \sqrt{-\Delta(t)} d t}\right],
$$

where $A_{I 2}=i(X-1 /(4 X)) A, A_{R 2}=(X+1 /(4 X)) A$, and $X=\exp \left(\tilde{k} \int_{r_{t 1}}^{r_{t 2}} \sqrt{\Delta(t)} d t\right)$. The first term of (32) is an incident wave toward $r_{t 1}$ since its group velocity $v_{g}=-\operatorname{Re}(\sqrt{-\Delta}) /(\tilde{k} R e(-s))$ is positive. However, its phase velocity $v_{\phi}=-\omega_{r} /(\tilde{k} R e(\sqrt{-\Delta}))$ is negative. The second term corresponds to the reflected wave but its amplitude $A_{R 2}$ is larger than the amplitude of the incident wave $A_{I 2}$. By inserting (32) in (8), we obtain the same asymptotic dispersion relation as (29) with the reflection coefficient

$$
\mathcal{R}=\mathcal{R}_{2} \equiv A_{R 2}^{2} / A_{I 2}^{2}=\mathrm{e}^{-i \pi}\left(\frac{4 X^{2}+1}{4 X^{2}-1}\right)^{2} .
$$

The formula (31) with $\mathcal{R}=\mathcal{R}_{2}$ therefore also applies to this case. Because of the over-reflection, the absolute value of the reflection coefficient $\left|\mathcal{R}_{2}\right|$ is now larger than unity so that the coefficient $\beta$ in (31) is complex and leads to the radiative instability. The stabilizing effect of the cyclonic background rotation on the radiative instability can be understood from (33). When $f$ increases from zero, the two turning points $r_{t 1}$ and $r_{t 2}$ move apart from each other, i.e., the potential barrier widens, implying that $X$ increases exponentially. Therefore, the reflection coefficient $\left|\mathcal{R}_{2}\right|$ tends to unity when $f$ increases meaning that the radiative instability is stabilized.

When $f$ is close to zero (i.e., $R o \gg 1$ ), the two turning points are close together so that the assumption of well-separated turning points is not valid. Instead, we can derive a local equation in the neighborhood of the critical point $r_{c}=R \sqrt{m \Omega / \omega}$ which is located in the middle between the two turning points $r_{t 1}$ and $r_{t 2}$. Around the critical point, we can approximate the Dopplershifted frequency $s$ and the function $\Delta$ as $s(r) \sim-\sqrt{\frac{4 \omega^{3}}{R^{2} m \Omega}} \epsilon \tilde{r}, \Delta(r) \sim \frac{4 \omega^{3}}{R^{2} m \Omega} \epsilon^{2}\left(\frac{R^{2}}{2 \omega^{2}} \frac{f}{\epsilon^{2}}-\tilde{r}^{2}\right)$ where $\tilde{r}=\frac{1}{\epsilon}\left(r-r_{c}\right)$ and $\epsilon=\left(\frac{R^{2} m \Omega}{4 \tilde{k}^{2} \omega^{3}}\right)^{1 / 4}$. Assuming that $f$ is small of order $f \sim O\left(\epsilon^{2}\right)$, (7) becomes at leading order in $\epsilon$,

$$
\frac{d^{2} p}{d \tilde{r}^{2}}-\frac{2 \tilde{r}}{\tilde{r}^{2}-a} \frac{d p}{d \tilde{r}}+\left(\tilde{r}^{2}-a+\frac{2}{\tilde{r}^{2}-a}\right) p(\tilde{r})=0,
$$

where $a=\frac{R^{2} f}{2 \omega^{2} \epsilon^{2}}=\frac{f \tilde{k} R}{\sqrt{m \Omega \omega}}$. The solution of (34) is

$$
p(\tilde{r})=b_{1} e^{\frac{i}{2} \tilde{r}^{2}}\left[U\left(i \frac{a}{4}-\frac{1}{4},-\frac{1}{2},-i \tilde{r}^{2}\right)+\frac{(i a-1)}{2} U\left(i \frac{a}{4}+\frac{3}{4}, \frac{1}{2},-i \tilde{r}^{2}\right)\right],
$$




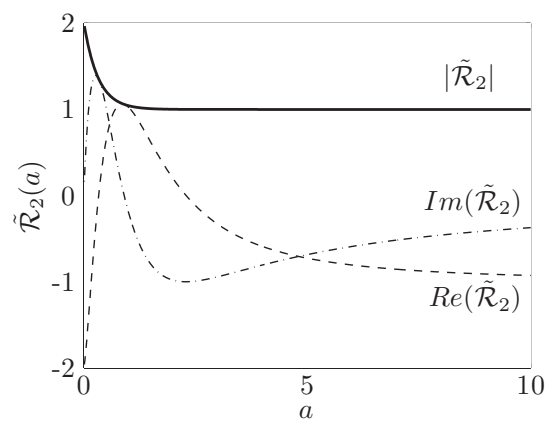

FIG. 9. Reflection coefficient $\tilde{\mathcal{R}}_{2}$ (37) as a function of $a$. The bold, dashed, and dashed-dotted lines are the absolute value, real part, and imaginary part, respectively.

where $U$ denotes the Kummer function ${ }^{19}$ and $b_{1}=\frac{A}{\sqrt{\tilde{k}_{c} \epsilon}} e^{\left(\frac{i \pi}{8}+\frac{a \pi}{8}\right)}\left(\frac{a}{4 e}\right)^{\frac{i a}{4}}$. By matching (35) to an outgoing wave for $\tilde{r} \rightarrow+\infty,{ }^{20}$ we find that the WKBJ approximation for $r<r_{t 1}$ which matches (35) for $\tilde{r} \rightarrow-\infty$, is the same as (32) except that $A_{I 2}$ and $A_{R 2}$ are replaced by

$$
\tilde{A}_{I 2}=A\left[\frac{-2 \pi e^{\left(\frac{i \pi}{4}+\frac{a \pi}{4}\right)}}{\Gamma\left(i \frac{a}{4}+\frac{5}{4}\right) \Gamma\left(-i \frac{a}{4}-\frac{1}{4}\right)}-1\right], \tilde{A}_{R 2}=A\left(i \frac{a}{4}\right)^{i \frac{a}{2}} e^{\frac{a}{2}(\pi-i)} \frac{2 \pi}{\Gamma\left(i \frac{a}{4}+\frac{5}{4}\right) \Gamma\left(i \frac{a}{4}-\frac{1}{4}\right)},
$$

respectively, where $\Gamma$ is the Gamma function. The asymptotic dispersion relations are then the same as (29) and (31) with

$$
\mathcal{R}=\tilde{\mathcal{R}}_{2}(a)=\tilde{A}_{R 2}^{2} / \tilde{A}_{I 2}^{2} .
$$

Figure 9 shows the reflection coefficient $\tilde{\mathcal{R}}_{2}$ as a function of $a=f \tilde{k} R / \sqrt{m \Omega \omega}$. We see that the reflection coefficient is $\left|\tilde{\mathcal{R}}_{2}\right|=2$ for $a=0$ (i.e., $f=0$ ) as obtained by Billant and Dizès. ${ }^{6}$ When $a$ increases, the reflection coefficient decreases quickly to unity. As mentioned before, this is because the size of the potential barrier $\delta r=r_{t 2}-r_{t 1} \approx 2 \epsilon \sqrt{a}$ increases. The wave over-reflection becomes exponentially small when $a \gtrsim 1$. Since the parameter $a$ is proportional to $f \tilde{k} / m$ (assuming $\omega \approx m \Omega$ ), this means that the radiative instability is stabilized as $f$ increases all the more rapidly than $\tilde{k}$ is large and $m$ is small.

\section{F. No turning point: Case (Ilb)}

Finally, when there is no turning point and $\Delta$ is positive, the WKBJ approximation (18) with $D=0$ is valid everywhere for $r>R$. The frequency at leading order is then

$$
\omega_{r}=m \Omega-\frac{\alpha}{R \tilde{k}},
$$

which is the same as (26) but the growth rate $\omega_{i}$ is zero. This configuration corresponds to the first branch when the Rossby number is smaller than $2 / m$, i.e., when (27) is not satisfied.

\section{G. Comparison with numerical results}

The various asymptotic formula derived in Subsections IV A-IV F are compared to the numerical results in Figure 10 for $m=1$ and $f / \Omega=[0,0.1,0.3]$. The WKBJ formula for the configurations with one turning point is shown by open circle for Eq. (26) (case I) and by crosses for Eqs. (29)(30) or squares for Eqs. (30)-(31) (case III). In each case, the agreement is good at large vertical wavenumber, for both the frequency and the growth rate (but only the case (I) is unstable). The dotted lines represent the predictions of the formula (29) together with the reflection coefficient (37) for two close turning points (case IIa). We see that the agreement is good except for the first branch because the double turning point is too close to the boundary $r=R$. Instead of the WKBJ formula 

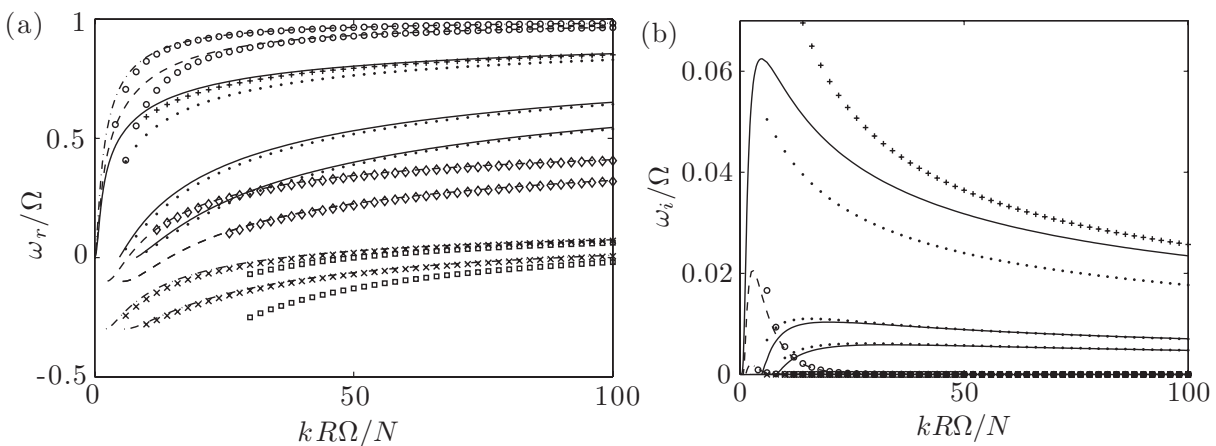

FIG. 10. Similar to Figure 1, but compared with the WKBJ asymptotic results also plotted with symbols: (•) two close turning points formula (29) using the reflection coefficient (37), (+) two close turning points formula with the Kummer function (35), (০) single turning point formula (26), ( $\diamond)$ two separate turning points formula (29) with the reflection coefficient (33), $(\times)$ single turning point formula (29), ( $\square$ ) single turning point formula (31).

(29) with (37), the solution (35) of the local equation (34) can be used directly in the dispersion relation (8) to circumvent this problem. This refined approximation is in better agreement for large $\tilde{k}$ as shown by the symbols + . The symbols $\diamond$ represent the asymptotic dispersion relation (29) with the reflection coefficient (33) for two well-separated turning points, a configuration prevailing for the next branches for $f / \Omega=0.1$. They are also in good agreements with numerical results.

The asymptotic dispersion relation (29) together with the reflection coefficient (37) for two close turning points is further tested in Figure 11 for the second branch for small background rotation. We see that the agreement is very good for large vertical wavenumber. It can be noticed that the growth rate is strongly reduced even if the Coriolis parameter is only of order $f / \Omega \sim O(0.01)$.

\section{ANTICYCLONIC ROTATION}

\section{A. Competition between the radiative and centrifugal instabilities}

We now investigate the effect of an anticyclonic rotation $(f<0)$. According to the Rayleigh criterion, ${ }^{21,22}$ the Rankine vortex is unstable to the centrifugal instability when $f$ is negative in the range $-2<f / \Omega<0$ (i.e., $R o<-1)$ since the Rayleigh discriminant $\phi=(f+\zeta)\left(2+2 U_{\theta} / r\right)$ is negative. However, we shall show that the radiative instability continues to exist when $f$ is slightly negative so that there is a competition between the radiative and centrifugal instabilities.

Figure 12 shows the growth rate of the first and second branches for $m=1$ for $f / \Omega=[0,-0.005$, $-0.01,-0.015]$. As soon as $f$ is non-zero, the growth rates increases with $\tilde{k}$ as $\tilde{k} \rightarrow \infty$ unlike for $f=0$ because of the centrifugal instability. However, the peak of the radiative instability at finite
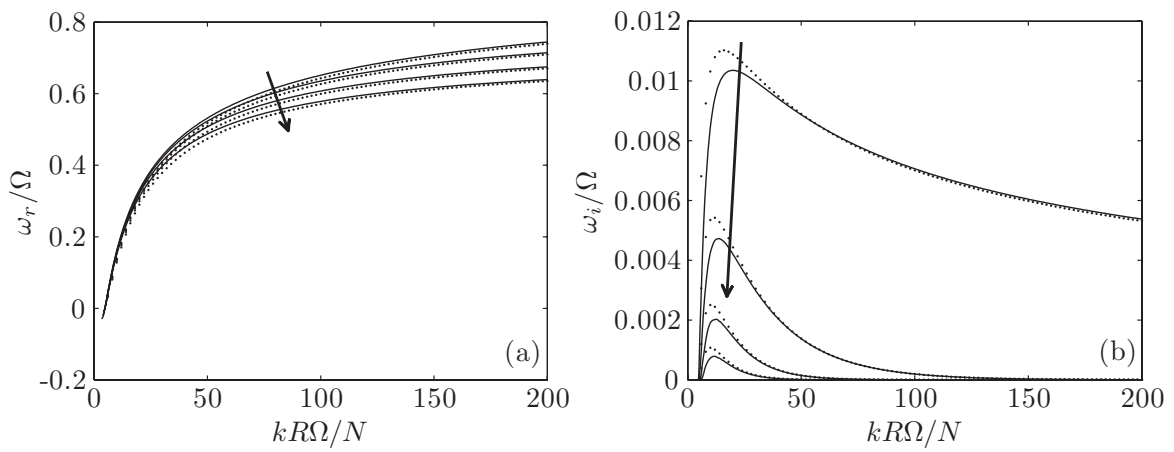

FIG. 11. (a) Frequency and (b) growth rate of the second branch for $m=1$ for $f / \Omega=[0,0.01,0.02,0.03]$. Solid and dotted lines are numerical and asymptotic results using the formula (29) and (37), respectively. $f$ increases in the direction of the arrows. 

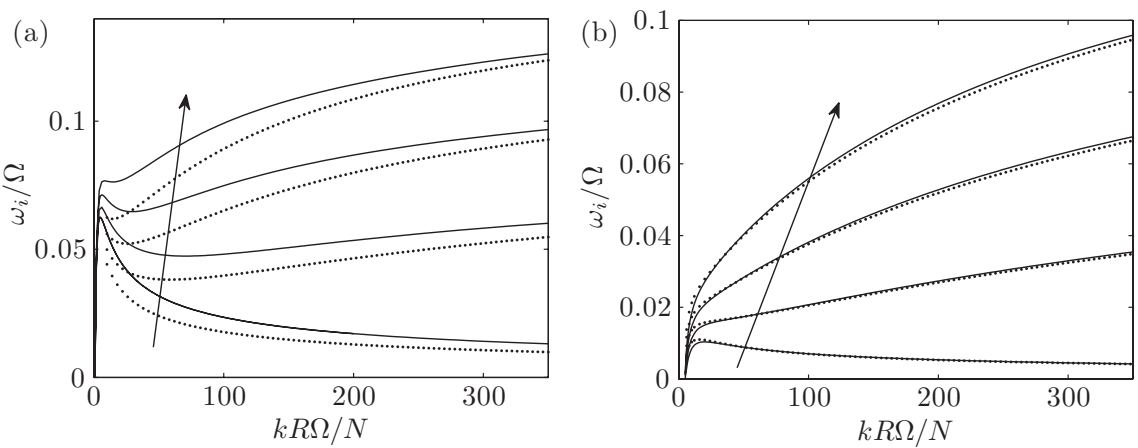

FIG. 12. Growth rate of (a) the first branch and (b) the second branch for $m=1$ for $f / \Omega=[0,-0.005,-0.01,-0.015]$. Solid and dotted lines represent numerical and asymptotic results using the formula (29) and (37), respectively. $f$ decreases from zero in the direction of the arrow.

$\tilde{k}$ remains present. In order to understand this behaviour, the local equation (34) around two close turning points and the resulting reflection coefficient $\tilde{\mathcal{R}}_{2}(37)$ can be used since they remain valid for $f<0$ provided that $-\frac{3 \pi}{2}<\arg (a)<\frac{\pi}{2}$. Dotted lines in Figure 12 represent the asymptotic growth rates obtained by inserting the reflection coefficient (37) into the asymptotic dispersion relation (29). They are in good agreements with the numerical results for all the values of $f$ investigated.

For very large $\tilde{k},(29)$ can be further expanded in powers of $\tilde{k}$ and gives the same result as (31) with $\mathcal{R}=\tilde{\mathcal{R}}_{2}$. Since $f$ is negative, there are now two imaginary terms in (31), the second term $\sqrt{f(f+2 \Omega)}$ which corresponds to the maximum growth rate of the centrifugal instability as $\tilde{k} \rightarrow \infty$,

$$
\omega_{i}=\sqrt{-f(f+2 \Omega)}
$$

and the last term involving $\beta$ which is responsible for the radiative instability and reaches a maximum at finite $\tilde{k}$ and decays to zero as $\tilde{k} \rightarrow \infty$. This proves that there is coexistence of the centrifugal and radiative instabilities.

In order to investigate the competition between these two instabilities, Figure 13 shows their maximum growth rates as a function of the inverse of the Rossby number. The growth rate peak at finite $\tilde{k}$ is associated with the maximum growth rate of the radiative instability, whereas the maximum growth rate of the centrifugal instability is $\sqrt{-f(f+2 \Omega)}$. The centrifugal instability becomes quickly the most dangerous instability for negative Ro but, interestingly, the radiative instability dominates the centrifugal instability when $R o \lesssim-400$.

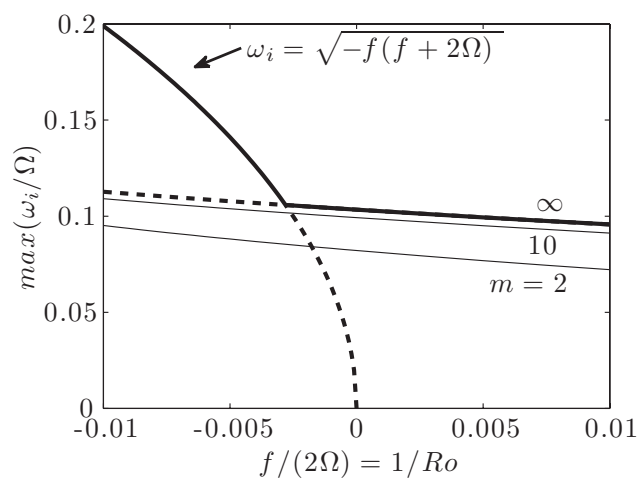

FIG. 13. Maximum growth rate of the radiative instability and the centrifugal instability as a function of the Rossby number $R o=2 \Omega / f$. Solid lines are numerical results of the maximum growth rate of the radiative instability and bold lines are maximum growth rates obtained from (14) for the radiative instability and from (39) for the centrifugal instability, respectively. 

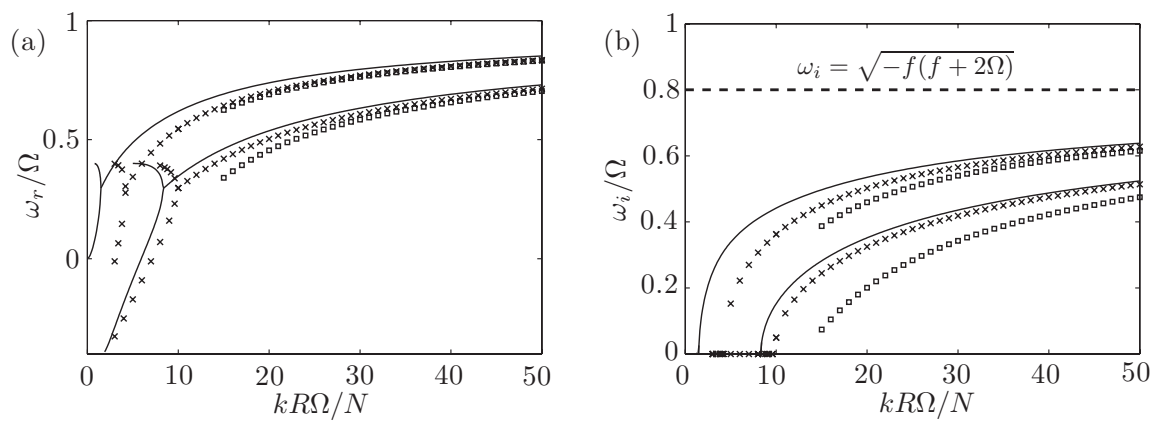

FIG. 14. (a) Frequency and (b) growth rate of the first two branches for $m=1$ for $f / \Omega=-0.4$. Solid lines are numerical results and symbols are asymptotic results as follows: $(\times)$ Eq. (29) and ( $\square)$ Eq. (43). The thick dashed line in (b) represents the maximum growth rate at $\tilde{k} \rightarrow \infty$.

\section{B. Results for $f / \Omega=O(-1)$}

We now investigate larger negative values of $f$ such that $f / \Omega=O(-1)$. Figure 14 shows a typical example of the frequency and growth rate for $m=1$ for $f / \Omega=-0.4$. The frequency of the first branch starts from $\omega_{r}=(m-1) \Omega=0$ at $\tilde{k}=0$ and increases with $\tilde{k}$, like for cyclonic rotation. However, there is now another branch which starts from $\omega_{r}=|f|$ at non-zero $\tilde{k}$ and decreases with $\tilde{k}$ (Figure 14(a)). Hence, these two branches meet at a finite wavenumber and the centrifugal instability appears above this wavenumber (Figure 14(b)). The same behaviour is observed for the next branches which starts from $\omega_{r}=|f|$ and $\omega_{r}=-|f|$ : the growth rate becomes positive when they meet. The numerical results for the centrifugal instability are compared to asymptotic results (symbols) obtained from a WKBJ analysis in the complex plane. Indeed, for non-zero azimuthal wavenumber, the growth rate and frequency of the centrifugal instability for large $\tilde{k}$ are comparable so that the function $\Delta(r)$ is complex on the real $r$-axis. Hence, we need to determine the location of the turning points in the complex plane and to consider the Stokes line defined by

$$
\operatorname{Re}\left(\int_{r_{t}}^{r} \sqrt{\Delta(t)} d t\right)=0
$$

When crossing a Stokes line, an exponentially growing WKBJ solution becomes exponentially decaying and vice versa. Hence, these lines delimit the regions of the complex plane where a given WKBJ approximation is valid. ${ }^{18,23,24}$ Three Stokes lines originate from a simple turning point.

Figure 15 shows an example of the configuration of turning points and the network of Stokes lines. There are always two turning points but as $f$ decreases from zero, one turning point $r_{t 2}$ moves

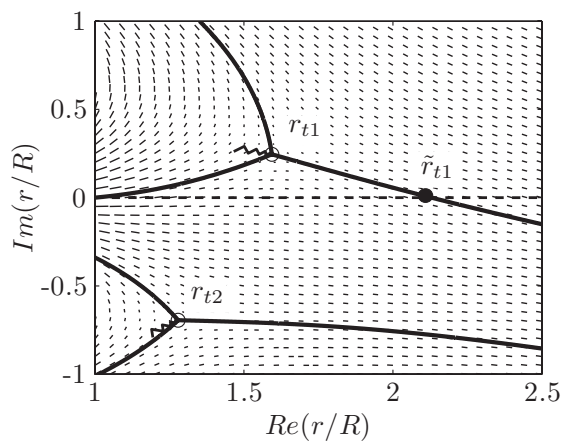

FIG. 15. Examples of the Stokes lines (heavy solid lines) network for $m=1$ and $k R \Omega / N=10$ for $f / \Omega=-0.1$ and $\omega / \Omega=0.412+0.140 i$. The dashed line and the symbols $\circ$ and $\bullet$ represent the real $r$-axis, the turning points $r_{t 1}, r_{t 2}$ and the point $\tilde{r}_{t 1}$ where the Stokes line emitted from $r_{t 1}$ meets the real $r$-axis, respectively. The zigzag lines show the branch cut. The short lines indicate the direction of the vectors $(\operatorname{Im}(\sqrt{\Delta}), \operatorname{Re}(\sqrt{\Delta}))$ which are parallel to the Stokes lines. 


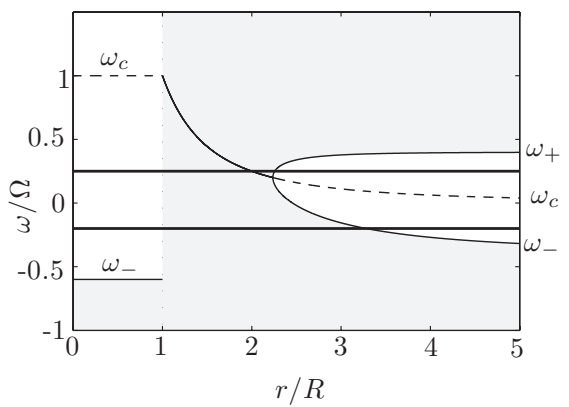

FIG. 16. Epicyclic frequencies $\omega_{+}$and $\omega_{-}$(thin solid lines) and critical frequency (thin dashed lines) as a function of $r$ for $f / \Omega=-0.4$ for $m=1$. Dotted lines represent the boundary of the vortex core. The two thick solid lines show examples of the frequency $\omega_{r}$ for the upper and lower neutral branches in Fig. 14. The regions where the WKBJ approximations are wavelike (i.e., $\Delta<0$ ) are shaded.

far away from the real axis and no Stokes line emanating from it cross the real axis when $|f|$ is sufficiently large (Figure 15(a)). Therefore, only the turning point $r_{t 1}$ which remains close to the real axis needs to be considered. Let $\tilde{r}_{t 1}$ denote the point where the Stokes line emitted from $r_{t 1}$ crosses the real axis. In order to construct an eigenmode, we choose the exponentially decaying solution for $r>\tilde{r}_{t 1}$

$$
p(r)=C \frac{\Delta^{1 / 4}}{r^{1 / 2}} \exp \left(-\tilde{k} \int_{r_{t 1}}^{r} \sqrt{\Delta} d t\right)
$$

The matching solution in the region $R<r<\tilde{r}_{t 1}$ is

$$
p(r)=C e^{\frac{i \pi}{4}} \frac{(-\Delta)^{1 / 4}}{r^{1 / 2}}\left[\exp \left(i \tilde{k} \int_{r}^{r_{t 1}} \sqrt{-\Delta} d t\right)-i \exp \left(-i \tilde{k} \int_{r}^{r_{t 1}} \sqrt{-\Delta} d t\right)\right],
$$

which is the same as (28). Inserting (42) in the dispersion relation (8) gives the same quantization condition as (29). From (29), we can further express the eigenfrequency in powers of $\tilde{k}$ at leading order

$$
\omega=m \Omega+i \sqrt{-f(f+2 \Omega)}-\frac{\beta^{\prime}}{(\tilde{k} R \Omega)^{2 / 3}}, \beta^{\prime}=i \frac{\left[\frac{3 \Omega^{2}}{\sqrt{2}}\left(n-\frac{1}{4}\right) \pi(-f-i m \sqrt{-f(f+2 \Omega)})\right]^{2 / 3}}{\sqrt{-f(f+2 \Omega)}},
$$

which is essentially the same as (31). As seen in Fig. 14, these asymptotic results predict well the frequency and growth rate of the centrifugal instability for large $\tilde{k}$. In particular, the bifurcations at low wavenumbers are well described by (29). For each branch, the bifurcation occurs at the vertical wavenumber where the turning point $r_{t 1}$ meets the real axis. For lower vertical wavenumbers, the turning point stays on the real axis and each branch separates into two branches with purely real frequency. As shown in the plot of the epicyclic frequencies (Figure 16), these branches correspond to neutral eigenmodes with a wavelike behaviour between the vortex core and the turning point. The bifurcation occurs around the frequency where the two epicyclic frequencies merge. The frequency of the upper branch then increases to $\omega=-f$ as $k$ decreases, while the frequency of the lower branch decreases as $k$ decreases. In the particular case of the axisymmetric mode $m=0$, the solution of (7) for $r>R$ can be found analytically as discussed in the Appendix.

For strong anticyclonic background rotation: $f \leq-2 \Omega$ (i.e., $-1 \leq R o<0$ ), the Rankine vortex is centrifugally stable but Park and Billant ${ }^{13}$ have found that the radiative instability also exists in this regime when the azimuthal wavenumber is larger than 2 . The properties of the radiative instability for large $m$ are very similar to those for cyclonic rotation. ${ }^{13}$ 


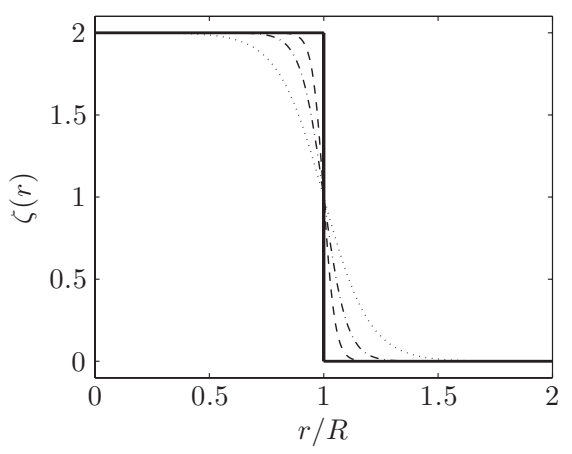

FIG. 17. Axial vorticity of the smoothed Rankine vortex (44) for $\delta=0$ (solid line), $\delta=0.05$ (dashed line), $\delta=0.1$ (dashed-dotted line), and $\delta=0.2$ (dotted line).

\section{STABILITY OF THE SMOOTHED RANKINE VORTEX}

The Rankine vortex is an idealized solution of the Euler equation with a discontinuity of vorticity. In order to investigate the stability of a vortex with a more realistic profile with continuous vorticity, we have considered the smoothed Rankine vortex proposed by Schecter and Montgomery ${ }^{8}$ with axial vorticity

$$
\zeta(r)=\Omega\left[1-\tanh \left(\frac{r / R-1}{\delta}\right)\right]
$$

where $\delta$ is a smoothness parameter. This profile tends to the Rankine vortex as $\delta \rightarrow 0$ and the vorticity jump becomes smoother as $\delta$ increases as illustrated in Figure 17. For this continuous profile, we use a classical shooting method to solve Eq. (7). ${ }^{8}$ The boundary condition at $r=0$ is $p(r) \sim r^{|m|}$.

Figure 18 shows the maximum growth rate $\max \left(\omega_{i}\right)$ as a function of the azimuthal wavenumber $m$ for different values of smoothness parameter $\delta$. While the maximum growth rate of the Rankine vortex is reached for $m \rightarrow \infty$, the maximum growth rate of the smoothed Rankine vortex occurs at finite azimuthal wavenumber $m_{\max }$ which decreases as $\delta$ increases. This behaviour is due to the presence of a critical point. The stabilizing effect of this critical point is related to the vorticity gradient $\left|\zeta^{\prime}\left(r_{c}\right)\right| \sim 1 / \cosh ^{2}(1 / m \delta){ }^{8}{ }^{8} 13$ Therefore, the damping rate is small when $m \delta \ll 1$, while it is large for $m \delta \gg 1$. This implies that the most amplified azimuthal wavenumber should scale like $m_{\max } \propto 1 / \delta$ as observed in Figure 18. This damping term is independent of $f / \Omega$ and thus it reduces the growth rate by approximately the same amount regardless of the values of $f / \Omega$ as seen in Figure 18.

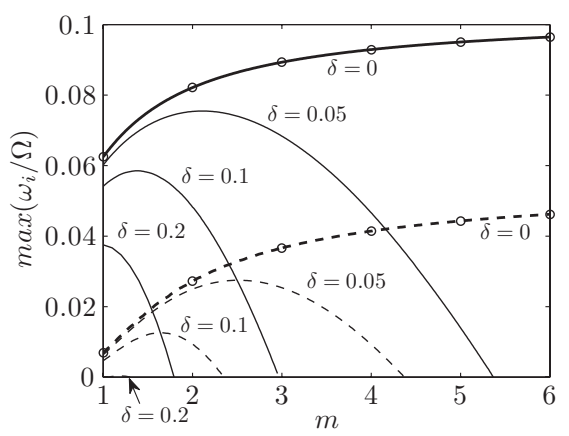

FIG. 18. Maximum growth rate $\max \left(\omega_{i}\right)$ of the first branch as a function of the azimuthal wavenumber $m$ for $\delta=[0,0.05$, $0.1,0.2]$ for $f / \Omega=0$ (solid lines) and $f / \Omega=0.2$ (dashed lines). Bold lines represent the limit $\delta=0$ (i.e., the Rankine vortex) 

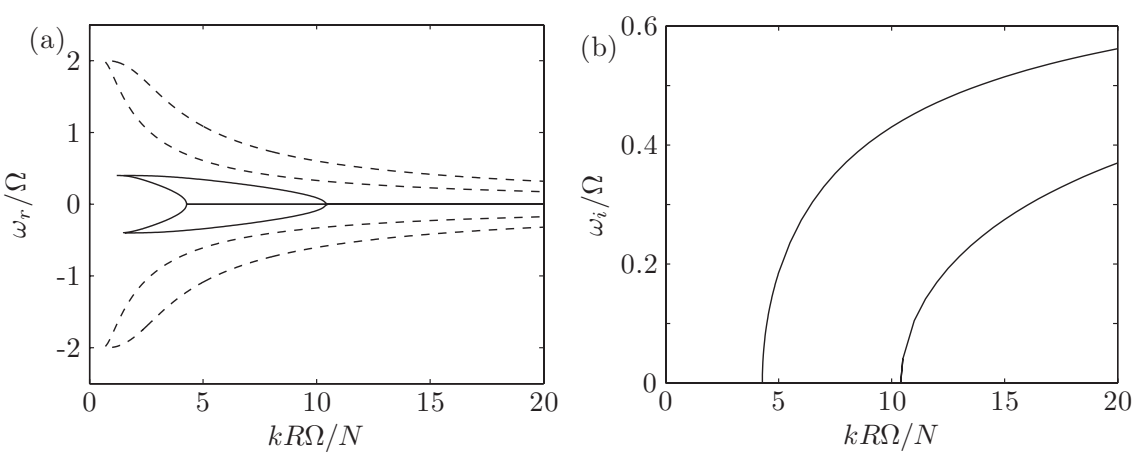

FIG. 19. (a) Frequency and (b) growth rate of the first two branches for $m=0$ as a function of the rescaled vertical wavenumber $k R \Omega / N$ for $f / \Omega=-0.4$ (solid lines), $f / \Omega=-2$ (dashed lines).

\section{CONCLUSIONS}

We have investigated the effect of the background rotation on the instabilities and waves sustained by a columnar vortex in a strongly stratified and rotating fluid. For the Rankine vortex, the radiative instability is stabilized in the presence of cyclonic background rotation and its maximum growth rate vanishes below a critical Rossby number which depends on $m: R o=2 / m$. The maximum growth rate $\omega_{i}$ increases with the azimuthal wavenumber $m$ and is about $\omega_{i} \sim 0.1 \Omega$ for $m \gg 1$ for $R o=\infty$ and exponentially small for $R o=O(1)$. The instability mechanism can be understood by means of a WKBJ analysis for large rescaled vertical wavenumber $k / N$. In the presence of cyclonic background rotation, the size of the potential barrier around the critical point is increased so that the over-reflection is reduced explaining the stabilization of the radiative instability. In some frequency ranges and below a critical Rossby number, there exist neutral waves. Some of them have a large frequency of order $O(|f|)$ and thereby are not captured by the quasi-geostrophic equation. In the presence of anticyclonic background rotation, the vortex is centrifugally unstable in the range $-2<f / \Omega<0$. However for slightly negative $f / \Omega \lesssim 0$ (i.e., $R o \lesssim-400$ ), the radiative instability is more unstable than the centrifugal instability. Unlike the Rankine vortex, the continuous smoothed Rankine vortex is most unstable at a finite azimuthal wavenumber which depends on the smoothness of the profile.

\section{ACKNOWLEDGMENTS}

This work was supported by the OLA (Oceanic LAyering) Project No. (ANR2011 Blanc SIMI 5-6 012-02).

\section{APPENDIX: CENTRIFUGAL INSTABILITY OF THE AXISYMMETRIC MODE: $\boldsymbol{m}=\mathbf{0}$} form $^{25}$

For $m=0$, the solution of (7) can be found analytically outside the vortex core $r>R$ in the

$$
p(r) \sim\left[\frac{1-v}{r} K_{v}\left(\tilde{k} r \sqrt{f^{2}-\omega^{2}}\right)-\tilde{k} \sqrt{f^{2}-\omega^{2}} K_{\nu-1}\left(\tilde{k} r \sqrt{f^{2}-\omega^{2}}\right)\right],
$$

where $K_{v}$ is the modified Bessel function of the second kind of order $v$ and $v^{2}=1+2 f R^{2} \Omega \tilde{k}^{2}$. The dispersion relation (8) can be simply expressed as

$\tilde{k} R \sqrt{(f+2 \Omega)^{2}-\omega^{2}} \frac{I_{0}\left(\tilde{k} R \sqrt{(f+2 \Omega)^{2}-\omega^{2}}\right)}{I_{1}\left(\tilde{k} R \sqrt{(f+2 \Omega)^{2}-\omega^{2}}\right)}+\tilde{k} R \sqrt{f^{2}-\omega^{2}} \frac{K_{v-1}\left(\tilde{k} R \sqrt{f^{2}-\omega^{2}}\right)}{K_{v}\left(\tilde{k} R \sqrt{f^{2}-\omega^{2}}\right)}+v-1=0$.

For cyclonic background rotation, the eigenmode which satisfies the outgoing wave boundary condition as $r \rightarrow \infty$ increases exponentially with $r$ (see also Billant and Le Dizès ${ }^{6}$ ). In contrast, for 
anticyclonic background rotation, there exist eigenmodes which decay as $r \rightarrow \infty$. In the range $-2 \Omega$ $<f<0$ (i.e., $R o<-1$ ), there is a centrifugal instability as shown in Sec. V.

Figure 19 shows the frequency and the growth rate for $f / \Omega=-0.4$ (centrifugally unstable) and $f / \Omega=-2$ (centrifugally stable). We see that there are two frequency branches: one starts from $\omega=f$ at non-zero $\tilde{k}$ and increases with $\tilde{k}$, while the other starts at $\omega=-f$ and decreases with $\tilde{k}$ (Figure 19(a)). For $f / \Omega=-0.4$, these frequency branches meet at a finite $\tilde{k}$ and become unstable for larger $\tilde{k}$ when they meet while for $f / \Omega=-2$, they do not meet and remains neutral whatever $\tilde{k}$ (Figure 19(b)).

${ }^{1}$ Lord Kelvin, "Vibrations of a columnar vortex," Philos. Mag. Ser. 5 10(61), 155-168 (1880).

${ }^{2}$ P. G. Saffman, Vortex Dynamics (Cambridge University Press, Cambridge, 1992).

${ }^{3}$ D. Fabre, D. Sipp, and L. Jacquin, "Kelvin waves and the singular modes of the Lamb-Oseen vortex," J. Fluid Mech. 551, 235-274 (2006).

${ }^{4}$ X. Riedinger, P. Meunier, and S. Le Dizès, "Instability of a vertical columnar vortex in a stratified fluid," Exp. Fluids 49, 673-681 (2010)

${ }^{5}$ X. Riedinger, S. Le Dizès, and P. Meunier, "Radiative instability of the flow around a rotating cylinder in a stratified fluid," J. Fluid Mech. 672, 130-146 (2011)

${ }^{6}$ P. Billant and S. Le Dizès, "Waves on a columnar vortex in a strongly stratified fluid," Phys. Fluids 21, 106602 (2009).

${ }^{7}$ S. Le Dizès and P. Billant, "Radiative instability in stratified vortices," Phys. Fluids 21, 096602 (2009).

${ }^{8}$ D. A. Schecter and M. T. Montgomery, "Damping and pumping of a vortex Rossby wave in a monotonic cyclone: Critical layer stirring versus inertia-buoyancy wave emission," Phys. Fluids 16, 1334 (2004).

${ }^{9}$ D. A. Schecter and M. T. Montgomery, "Conditions that inhibit the spontaneous radiation of spiral inertial-gravity waves from an intense mesocale cyclone," J. Atmos. Sci. 63, 435 (2006).

${ }^{10}$ D. A. Schecter, "The spontaneous imbalance of an atmospheric vortex at high rossby number," J. Atmos. Sci. 65, 2498 (2008).

${ }^{11}$ R. Ford, "The instability of an axisymmetric vortex with monotonic potential vorticity in rotating shallow water," J. Fluid Mech. 280, 303-334 (1994).

${ }^{12}$ E. Broadbent and D. W. Moore, “Acoustic destabilization of vortices,” Philos. Trans. R. Soc. London, Ser. A 290, 353 (1979).

${ }^{13}$ J. Park and P. Billant, "Radiative instability of an anticyclonic vortex in a stratified rotating fluid," J. Fluid Mech. 707, 381-392 (2012).

${ }^{14}$ W. D. Smyth and J. C. McWilliams, "Instability of an axisymmetric vortex in a stably stratified, rotating environment," Theor. Comput. Fluid Dyn. 11, 305-322 (1998).

${ }^{15}$ T. Miyazaki and H. Hanazaki, "Baroclinic instability of Kirchhoff's elliptic vortex," J. Fluid Mech. 261, 253-271 (1994).

${ }^{16}$ S. Le Dizès and X. Riedinger, "The strato-rotational instability of Taylor-Couette and Keplerian flows," J. Fluid Mech. 660, 147-161 (2010).

${ }^{17}$ S. Le Dizès and L. Lacaze, “An asymptotic description of vortex Kelvin modes,” J. Fluid Mech. 542, 69-96 (2005).

${ }^{18}$ C. M. Bender and S. A. Orszag, Advanced Mathematical Methods for Scientists and Engineers (McGraw-Hill, New York, 1978).

${ }^{19}$ M. Abramowitz and I. A. Stegun, Handbook of Mathematical Functions (Dover, New York, 1965).

${ }^{20}$ L. Parras and S. Le Dizès, "Temporal instability modes of supersonic round jets," J. Fluid Mech. 662, 173-196 (2010).

${ }^{21}$ Lord Rayleigh, "On the dynamics of revolving fluids," Proc. R. Soc. London 93, 148-154 (1917).

${ }^{22}$ R. C. Kloosterziel and G. J. F. van Heijst, "An experimental study of unstable barotropic vortices in a rotating fluid," J. Fluid Mech. 223, 1-24 (1991).

${ }^{23}$ F. W. J. Olver, Asymptotics and Special Functions (Academic Press, New York, 1974).

${ }^{24} \mathrm{P}$. Billant and F. Gallaire, "Generalized Rayleigh criterion for non-axisymmetric centrifugal instabilities," J. Fluid Mech. 542, 365-379 (2005).

${ }^{25}$ A. Lazar, A. Stegner, and E. Heifetz, "Inertial instability of intense stratified anticyclones. Part I : Generalized stability criterion," J. Fluid Mech. (in press). 